ARTICLE OPEN

\title{
Dynamic synthesis of Heisenberg-limited spin squeezing
}

\author{
Long-Gang Huang ${ }^{1}$, Feng Chen (D) ${ }^{1}$, Xinwei $\mathrm{Li}^{1}$, Yaohua Li ${ }^{1}{ }^{1}$, Rong Lü ${ }^{1,2}$ and Yong-Chun Liu (D) ${ }^{1,2 凶}$
}

Spin squeezing is a key resource in quantum metrology, allowing improvements of measurement signal-to-noise ratio. Its generation is a challenging task because the experimental realization of the required squeezing interaction remains difficult. Here, we propose a generic scheme to synthesize spin squeezing in non-squeezing systems. By using periodical rotation pulses, the original non-squeezing interaction can be transformed into squeezing interaction, with significantly enhanced interaction strength. The sign of the interaction coefficient is also flippable, facilitating time-reversal readout protocol for nonlinear interferometers. The generated spin squeezing is capable of achieving the Heisenberg limit with measurement precision $\propto 1 / N$ for $N$ particles and its robustness to noises of pulse areas and separations has been verified as well. This work offers a path to extending the scope of Heisenberg-limited quantum precision measurements in non-squeezing systems.

npj Quantum Information (2021)7:168; https://doi.org/10.1038/s41534-021-00505-z

\section{INTRODUCTION}

Squeezed spin states ${ }^{1-4}$ are such entangled states of collective spins in which the uncertainty of one spin component perpendicular to the mean spin direction is reduced below the standard quantum limit (SQL). They have attracted broad attention due to their applications in high-precision measurements ${ }^{5-10}$ and in the study of many-body entanglement ${ }^{11-17}$. Over the past few decades, many studies have been proposed to realize spin squeezing ${ }^{4}$, mainly involved in Bose-Einstein condensates (BECs) with atom-atom collisions ${ }^{8,9,18-20}$, atom-light interaction systems ${ }^{21-25}$

To generate spin squeezing, one direct method is to transfer the squeezing from squeezed light to atomic ensembles, but the transfer efficiency is limited and the degree of spin squeezing depends on the performance of light ${ }^{26-29}$. Quantum nondemolition (QND) measurement is also a feasible method to realize spin squeezing, but the acquired squeezing is not deterministic and is degraded by the performance of the detector ${ }^{30-40}$. Another major type of method to deterministically generate spin squeezing is utilizing squeezing interactions, which typically include the one-axis twisting (OAT) and two-axis twisting (TAT) interactions $s^{1,4,41-47}$. The OAT interaction, which can be experimentally implemented in some systems including $B E C s^{8,9,48}$, trapped ions $s^{49-51}$ and superconducting qubits $^{52-54}$, reaches a squeezing degree $\propto 1 / N^{2 / 3}$ for $N$ particles. As for the TAT interaction, which is mainly reported in theoretical scheme $\mathrm{s}^{46,55,56}$, the squeezing degree is $\propto 1 / \mathrm{N}$, approaching to the Heisenberg limit $(\mathrm{HL})$, but implementation of TAT squeezing still remains a challenge. In the atom-light interaction systems, the double-pass squeezing approach based on the Faraday magnetooptical rotation is proposed ${ }^{57}$, but the squeezing performance $\propto 1 /$ $N^{2 / 5}$ is far from being satisfactory, which is due to the unwanted residual atom-light entanglement ${ }^{58,59}$.

Here, we propose a dynamic synthesis scheme to deterministically generate high-degree spin squeezing, without requiring original squeezing interactions. Based on the common nonsqueezing interactions, denoted by equation (1), and a periodic rotation pulse sequence, the squeezing interactions can be dynamically synthesized, facilitating a squeezed spin state from an initially uncorrelated state. Both OAT and TAT interactions can be effectively synthesized, which hence leads to a squeezing performance approaching to the HL. Our scheme hence largely enriches the variety of systems that can realize spin squeezing. Furthermore, in our scheme, the strength of the generated interaction is enhanced (faster dynamics or shorter evolution) and the sign of the interaction coefficient is flippable, presenting its capability of realizing the time-reversal dynamics. It hence gives rise to a potential platform to realize technical-noise-immune nonlinear interferometer and study the quantum chaos and information scrambling by measuring the out-of-time-order correlator ${ }^{60-65}$.

\section{RESULTS}

\section{System model}

We consider a universal model with the following interaction Hamiltonian

$H_{\text {int }}=g S_{z} J_{z}$,

where $g$ denotes the coupling strength between two subsystems described by the collective spins $\mathbf{S}$ and $\mathbf{J}$. This Hamiltonian commonly exists in various systems, such as atom-light interaction systems $\mathrm{s}^{31,37}$ and dipole-dipole interaction systems ${ }^{66}$, as illustrated in Fig. 1(a), (b). Note that in the split BEC system ${ }^{66}$, there exist intra-species interactions $S_{z}^{2}$ and $J_{z}^{2}$ apart from inter-species interaction $S_{z} J_{z}$, while in the system of cold or thermal atoms, the intra-species interactions vanish and only inter-species interaction between two subsystems is left, which is the form of equation (1). In the context of atomic ensembles, the operators $S_{\mu}=\sum_{k=1}^{N_{s}} \sigma_{\mathrm{S}, \mu}^{(k)} / 2$ and $J_{\mu}=\sum_{k=1}^{N_{j}} \sigma_{\mathrm{J}, \mu}^{(k)} / 2(\mu=x, y, z)$ define the collective spin components for the two subsystems, with $\sigma_{S, \mu}^{(k)}$ and $\sigma_{J, \mu}^{(k)}$ being the corresponding Pauli matrices for the $k$-th spin- $1 / 2$ (or pseudospin) particle, respectively. As for atom-light interaction system, the operators $J_{\mu}$ denote the Stokes operators, which are related to the differences between the number operators of the photons polarized in different orthogonal bases (see Supplementary Note A). Both of them satisfy the usual $S U(2)$ angular momentum commutation relations $\left[S_{i}, S_{j}\right]=i \varepsilon_{i j k} S_{k}$ and $\left[J_{i}, J_{j}\right]=$ i $\varepsilon_{i j k} J_{k}$, where $\varepsilon_{i j k}$ is the Levi-Civita symbol. 
(a)

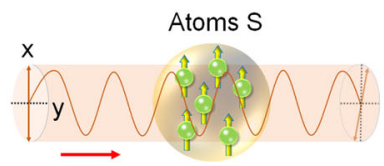

Light J

(b)

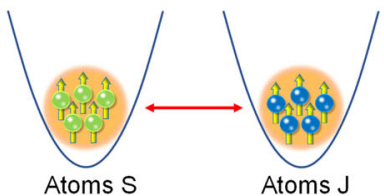

(c)

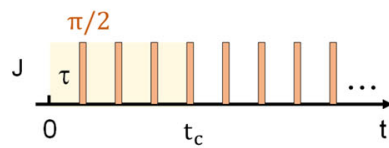

(d)

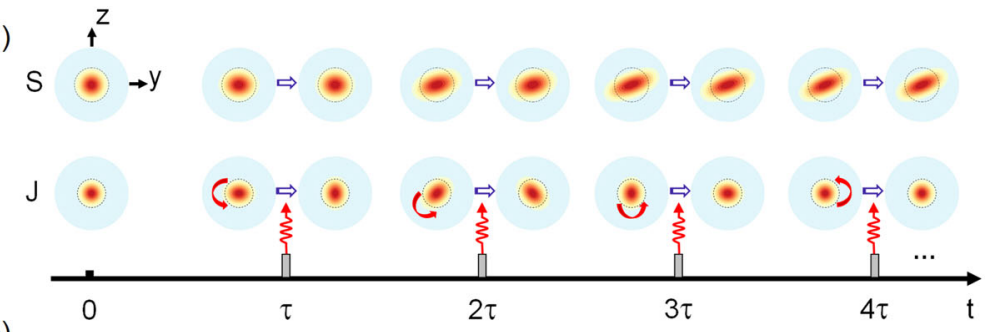

(e)

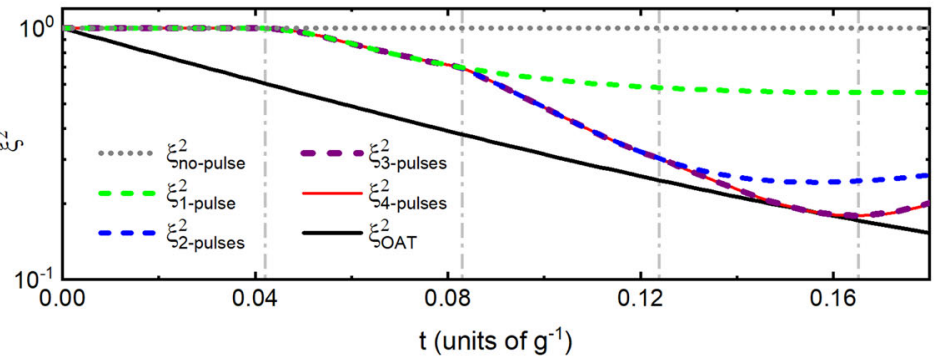

Fig. 1 Spin squeezing via dynamic synthesis. a Schematic diagram of atom-light interaction system with Faraday magneto-optic rotation. b Schematic diagram of dipole-dipole interaction system consisting of two atomic ensembles. c An illustration of the pulse sequence for the synthesis of the OAT squeezing. Each period $t_{c}$ contains four $\pi / 2$ rotation pulses (orange rectangles) with identical pulse separation $\tau$, imposed on subsystem $J$. d Evolution of the quantum states for subsystems $S$ (upper panel) and $J$ (lower panel) during the first pulse cycle, represented by the Husimi Q function on the generalized Bloch spheres. The dashed line circles indicate the outline of the initial coherent spin state for $S$ and $J$, respectively. e Evolution of the squeezing parameters $\xi^{2}$ for subsystem $S$ when different number of rotation pulses are applied. Gray dotted curve, no pulse; green dashed curve, 1 pulse applied at $t=\tau$; blue dashed curve, 2 pulses applied at $t=\tau$, $2 \tau$; purple dashed curve, 3 pulses applied at $t=\tau, 2 \tau, 3 \tau$; red solid curve, 4 pulses applied at $t=\tau, 2 \tau, 3 \tau$, $4 \tau$. The black solid curve denotes the results of the effective OAT interaction (5), and the vertical gray dot-dashed lines mark time instants for $t=\tau, 2 \tau, 3 \tau, 4 \tau$. The particle numbers are $N_{\mathrm{s}}=40$ and $N_{\mathrm{j}}=60$, and the pulse separation is $\tau=0.041\left(g^{-1}\right)$.

To be clear, we clarify that the studied spin squeezing is focused on one subsystem (e.g., S), which contains the contribution of the other subsystem $J$ through their interaction. This is mainly based on the fact that the squeezing of one subsystem is usually easier to be implemented than that of the united system $(S+J)$ in practical application, especially for the case with quite different subsystems. For example, for atom-light interaction systems, the polarization of photons and collective atomic spin can not be naturally combined to serve as the whole resources of squeezing (see Supplementary Note $H$ ). More specifically, in the model Hamiltonian (1), intra-species interaction $\left(S_{z}^{2}\right.$ or $\left.J_{z}^{2}\right)$ in both subsystems is absent and the atoms hence maintain approximately uncorrelated following the evolution based on the interaction Hamiltonian (1), if they are initially uncorrelated. An effective intraspecies interaction always occurs as a high-order effect from the trivial inter-species interaction $\left(S_{z} J_{z}\right)$, i.e., subsystem $J$ carries the interaction among atoms in subsystem $S$, as an auxiliary force carrier.

To amplify such a high-order effect and eliminate the influence of low-order interaction, we put forward a controlled pulse scheme, which contains several rotation pulses described by the following Hamiltonian

$H_{\text {rot }}=\Omega_{1}(t) J_{x}+\Omega_{2}(t) S_{x}$.

It leads to a spin rotation of angle $\theta_{1,2}=\int_{t_{0}}^{t_{0}+\Delta t} \Omega_{1,2}(t) d t$ around $x$ axis over a short pulse duration $\Delta t$ starting from $t_{0}$ for subsystem $J$ and $S$, respectively. Their corresponding rotation operators are defined as $R_{\mathrm{J}}(\theta)=\mathrm{e}^{-\mathrm{i} \theta J_{\mathrm{x}}}$ and $R_{\mathrm{S}}(\theta)=\mathrm{e}^{-\mathrm{i} \theta S_{x}}$.

\section{Generation of effective OAT squeezing}

First, we propose a pulse scheme to synthesize the effective OAT squeezing. In this case, only periodic $\pi / 2$ rotation pulses are required to implement on the subsystem $J$ with equal pulse separation $\tau$. Each period contains four segments of free evolution and four $\pi / 2$ rotation pulses, as sketched in Fig. 1(c). Neglecting the pulse duration $\Delta t$, the evolution operator for a single period $t_{\mathrm{c}} \simeq 4 \tau$ is given by

$U_{1}(4 \tau)=\left[R_{J}\left(\frac{\pi}{2}\right) U(\tau)\right]^{4}$,

where $U(\tau)=\mathrm{e}^{-\mathrm{i} g S_{z} J_{z} \tau}$ is the free evolution operator under the interaction Hamiltonian (1). The evolution operator can be rewritten as

$$
U_{1}(4 \tau)=\mathrm{e}^{\mathrm{i} g S_{z} J_{y} \tau} \mathrm{e}^{\mathrm{i} g S_{z} J_{z} \tau} \mathrm{e}^{-\mathrm{i} g S_{z} J_{y} \tau} \mathrm{e}^{-\mathrm{i} g S_{z} J_{z} \tau} \equiv \prod_{k=1}^{4} \mathcal{U}_{k},
$$

as a product of four unitary operators. According to the BakerCampbell-Hausdorff formula, omitting the higher-order terms $\mathcal{O}\left((g \tau)^{2}\right)$, the evolution operator is approximately derived as $U_{1}(4 \tau) \simeq \exp \left(-\mathrm{i} g^{2} S_{z}^{2} J_{x} \tau^{2}\right)$. When the subsystem $J$ initially stays in a coherent state ( $N_{\mathrm{j}}$ uncorrelated particles) polarized along the $x$ axis, i.e., the eigenstate of $J_{x}$ with the eigenvalue $N_{j} / 2$, the effective Hamiltonian can be obtained as (see Supplementary Note B),

$H_{\text {eff }}^{\mathrm{OAT}}=X_{\mathrm{eff}} S_{Z}^{2}$,

$X_{\text {eff }}=\frac{1}{8} N_{\mathrm{j}} g^{2} \tau$

We hence obtain an effective OAT Hamiltonian, giving rise to squeezing dynamics in subsystem $S$.

The understanding of the pulse scheme is as follows. Subsystem $J$ plays the role of an intermediary to induce the intra-species interaction in subsystem $S$. This process is analogous to the effective electron-electron interaction mediated by the phonons (lattice vibrations) in condensed matter physics, such as the superconducting Bardeen-Cooper-Schrieffer theory, which can be treated by the Frohlich-Nakajima transformation ${ }^{67,68}$. The product of the first three evolution operators, $\mathcal{U}_{3} \mathcal{U}_{2} \mathcal{U}_{1}$, in each period constitutes an equivalent Frohlich-Nakajima transformation $(\mathrm{FNT})^{67,68}$, representing that we apply unitary transformation on the evolution operator. According to the operator expansion 
formula

$\mathrm{e}^{S} H \mathrm{e}^{-S}=H+[S, H]+\frac{1}{2 !}[S,[S, H]]+\ldots$,

the product $\mathcal{U}_{3} \mathcal{U}_{2} \mathcal{U}_{1}$ can be expanded as

$\mathrm{e}^{S} \mathrm{e}^{-\mathrm{i} H \tau} \mathrm{e}^{-S}=\mathrm{e}^{-\mathrm{i}\left\{H+[S, H]+\frac{1}{2 !}[S,[S, H]]+\ldots\right\} \tau}$,

where $H=g S_{z} J_{y}$ denotes the Hamiltonian in the second segment in each period and $S=i g S_{z} J_{z} \tau$ denotes the unitary transformation, arising from the real unitary evolution. The proper form of $S$ can make the commutator $[S, H]$ contains squeezing term $g^{2} \tau J_{x} S_{z}^{2}$ leading to the squeezing of subsystem $S$. In order to eliminate the first-order term, i.e., the residual linear interacting term in equation (8), we need to add another segment of real unitary evolution $e^{\mathrm{i} H \tau}$, i.e., $\mathcal{U}_{4}$, such that

$$
\mathrm{e}^{\mathrm{i} H \tau} \mathrm{e}^{S} \mathrm{e}^{-\mathrm{i} H \tau} \mathrm{e}^{-S}=\mathrm{e}^{-\mathrm{i}\left\{[S, H]+\frac{1}{2 !}[S,[S, H]]+\ldots\right\} \tau} \approx \mathrm{e}^{-\mathrm{i} g^{2} \tau^{2} J_{x} S_{z}^{2}},
$$

for which the higher-order terms are ignored. This corresponds to the evolution operator of squeezing interaction, indicating that the second-order perturbation leads to the OAT squeezing interaction $S_{z}^{2}$. In each period, subsystem $J$ hence serves as an auxiliary system for "information storage, processing and feedback", which stores the quantum state of subsystem $S$, and transfers the information back to subsystem $S$. (see Supplementary Note $D$ for details). It is noticed that no additional information about subsystem $S$ is carried by subsystem $J$ after each complete period, since the output state of $J$ in each period remains an uncorrelated coherent spin state the same as its input, as shown in Fig. 1 (d). Note that in the reported cavity feedback mechanism ${ }^{7,69}$, the cavity field plays the similar role of subsystem $J$ in our scheme. However, we should point out that our scheme is general to extensive systems as long as the interaction has the form of coupling two collective (pseudo-)spins, rather than being limited in the specific atom-light interaction systems.

To verify the validity of the effective OAT Hamiltonian (5), we numerically investigate the evolutions of the quantum state in the full Hilbert space within the first period of our scheme. It is demonstrated by their representations (Husimi Q function after partially tracing out one of the subsystems) on the generalized Bloch spheres in Fig. 1(d). The overall quasiprobability distribution of subsystem $J$ rotates 90 degrees after each $\pi / 2$ pulse. While as for subsystem $S$, it is continuously squeezed, representing the OAT dynamics. The degree of spin squeezing is usually quantified by the squeezing parameter $\xi^{2}=4\left(\Delta S_{\perp}\right)_{\min }^{2} / N_{s}$ for Kitagawa and Ueda criterion ${ }^{1}$, or $\xi_{R}^{2}=N_{s}\left(\Delta S_{\perp}\right)_{\min }^{2} /|\langle\mathbf{S}\rangle|^{2}$ for Wineland criterion $^{2,3}$, where $\left(\Delta S_{\perp}\right)_{\min }^{2}$ is the minimum of the fluctuation $\left(\Delta S_{\perp}\right)^{2}=$ $\left\langle S_{\perp}^{2}\right\rangle-\left\langle S_{\perp}\right\rangle^{2}$ for the spin component perpendicular to the mean spin direction and $|\langle\mathbf{S}\rangle|$ is the mean spin length. In Fig. 1(e) the evolution of the squeezing parameters for different cases are exhibited. Without rotation pulses, the squeezing parameter keeps $\xi^{2}=1$ (gray dotted curve), denoting that the $S_{z} J_{z}$ type interaction alone cannot dynamically introduce spin squeezing starting from initially uncorrelated states as we previously discussed. When rotation pulses are applied, the squeezing parameter decreases, indicating spin squeezing. At the end of the first period with four $\pi / 2$ rotation pulses, the squeezing parameter (red solid curve) touches the curve for the OAT squeezing (black solid curve) governed by the Hamiltonian (5). It is noted that only one or two rotation pulses can also lead to squeezing (dashed curves), while the squeezing degree cannot reach as high as the OAT results.

The optimal squeezing parameter (for Kitagawa and Ueda criterion) and the corresponding squeezing time for the synthesized effective OAT interaction are derived as

$\xi_{\min }^{2} \simeq \frac{1}{2}\left(\frac{N_{\mathrm{s}}}{3}\right)^{-\frac{2}{3}}, t_{\min } \simeq \frac{8 \times 3^{1 / 6}}{g^{2} \tau N_{\mathrm{j}} N_{\mathrm{s}}^{2 / 3}}$.
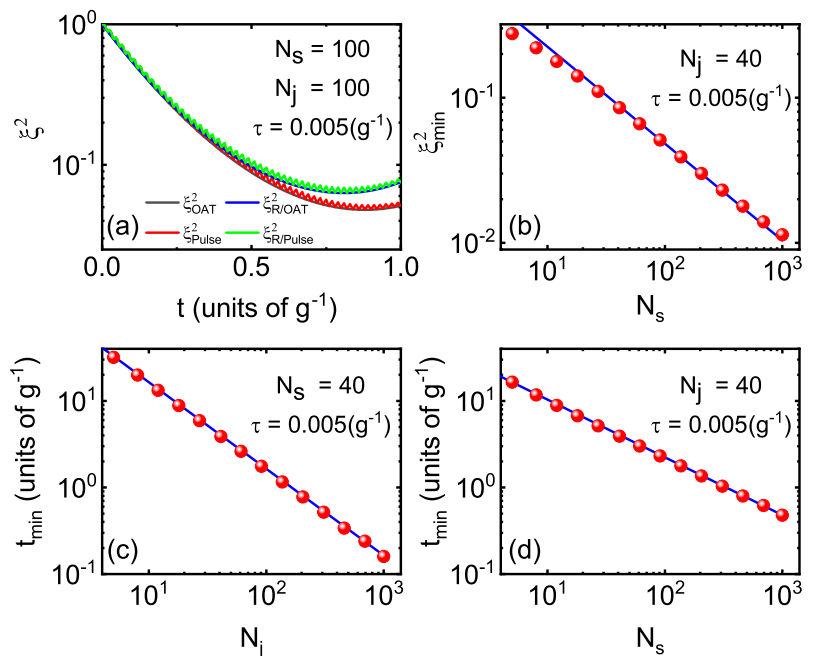

Fig. 2 Dynamic synthesis of OAT squeezing. a Evolution of the spin squeezing parameter $\xi^{2}$ for our pulse scheme (red oscillating curve: criterion of Kitagawa and Ueda'; green oscillating curve: criterion of Wineland ${ }^{2,3}$ ) and for the corresponding effective OAT Hamiltonian (5) (black and blue solid curves for the two parameters). b The optimal spin squeezing parameter $\xi_{\min }^{2}$ for Kitagawa and Ueda criterion as a function of particle number $N_{\mathrm{s}}$ for the pulse scheme (red solid balls). $\mathbf{c}$ and $\mathbf{d}$ The optimal squeezing time $t_{\min }$ as a function of particle numbers $N_{\mathrm{j}}$ and $N_{\mathrm{s}}$ for the pulse scheme (red solid balls), respectively. The blue solid curves in b, $\mathbf{c}$ and $\mathbf{d}$ are predicted by equation (10). The parameters are given in each subgraph.

Their validity for both Kitagawa parameter $\xi^{2}$ and Wineland parameter $\xi_{R}^{2}$ is demonstrated by the exact numerical simulation as compared in Fig. 2, including the evolution of squeezing parameter and the $N$ scaling about the optimal squeezing parameter and squeezing time. The power-law scalings of both $\xi_{\min }^{2}$ and $t_{\min }$ with respect to subsystem $S$ are the same as the OAT case, i.e., $\xi_{\min }^{2} \propto 1 / N_{\mathrm{s}}^{2 / 3}$ and $t_{\min } \propto 1 / N_{\mathrm{s}}^{2 / 3}$. Here we consider a fixed $\tau$, since it is an artificially controlled parameter, while the restricted condition for $\tau$, i.e., Eq. (32) in Supplementary Note C, is just a sufficient condition. It notes that the optimal squeezing parameter for subsystem $S$ is independent of subsystem $J$, since they become uncorrelated after each pulse sequence period. As for the optimal squeezing time, there is an additional power-law scaling with respect to $N_{\mathrm{j}}$, i.e., $t_{\min } \propto 1 / N_{\mathrm{j}}$, as shown in Fig. 2(c), due to the dependence of $N_{\mathrm{j}}$ on the effective coupling strength. It suggests that an additional degree of freedom to accelerate the arrival of optimal spin squeezing is available, resulting in preceding the conventional OAT squeezing that is usually limited in a single atomic ensemble. In addition, the squeezing of one subsystem for our scheme performs better than that of the united system under free evolution of the Hamiltonian (1), when the particle numbers of two subsystems are quite different (see Supplementary Note $\mathrm{H}$ ).

Furthermore, our scheme is also utilizable for a fairly small number of pulses. A large number of pulses, corresponding to sufficiently small pulse separation $\tau$, can guarantee the validity of our pulse scheme almost all the time, as shown in Fig. 3(a), which takes the effective OAT scheme with 200 pulses as an example. However, if we only focus on achieving the optimal squeezing, the pulse separation is not necessary to be tiny, and thus the pulse number can be reduced. As plotted in Fig. 3(b)-(d), if we reduce the pulse number, the oscillation amplitude of the squeezing parameter increases, but the optimal squeezing is still achievable. This is because only the time instants at the end of each period are important. From Fig. 3(d) we can find that even for as few as 4 pulses, it is sufficient to reach the optimal squeezing. Moreover, 
4
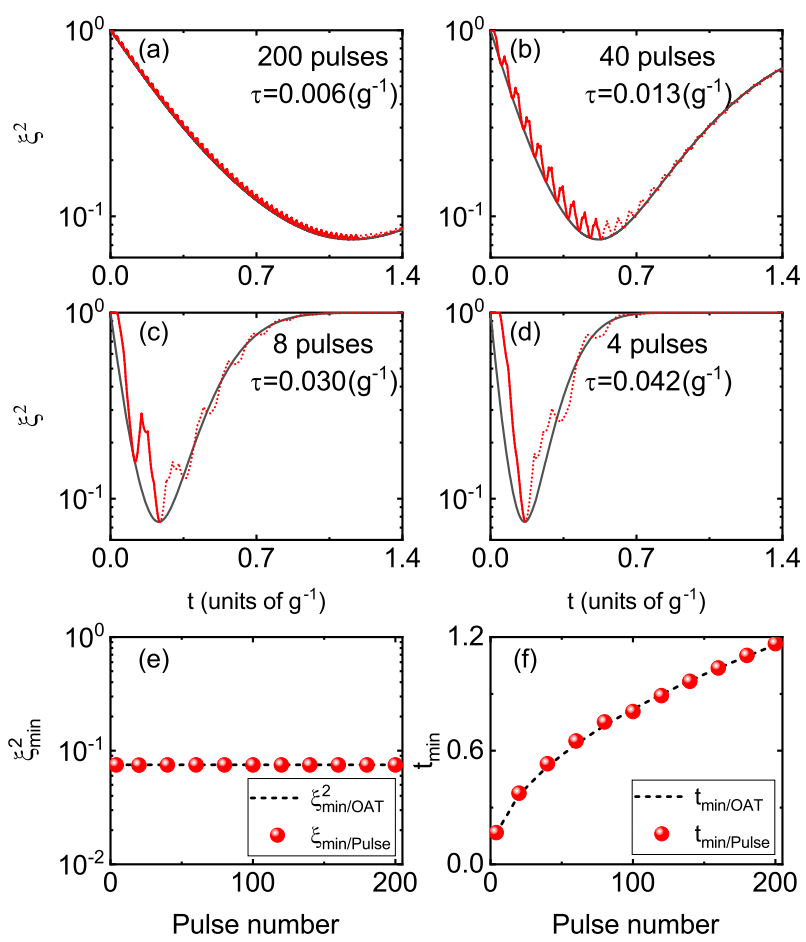

Fig. 3 Influence of pulse numbers on the squeezing parameter $\boldsymbol{\xi}^{2}$. a-d Evolution of $\xi^{2}$ for different pulse numbers (with corresponding values of $\tau$, obtained from Eq. (35) in Supplementary Note C) 200, 40, 8 and 4 that are applied before reaching the optimal spin squeezing (red solid curves), compared with the effective OAT dynamics (black solid curves). The red dashed curves correspond to the results when we continue to apply the pulse sequences after reaching the optimal squeezing. $e$ and $\mathbf{f}$ The optimal spin squeezing parameter $\xi_{\min }^{2}$ and the optimal squeezing time $t_{\min }$ as functions of pulse numbers (four $\pi / 2$ pulses in a period) for the pulse scheme (red solid balls). The black dashed curves denote the effective OAT dynamic results for comparison. The particle numbers are $N_{\mathrm{s}}=50$ and $N_{\mathrm{j}}=$ 100.

fewer pulse number (larger pulse separation $\tau$ ) corresponds to stronger interaction strength, and thereby shorter squeezing time, as indicated from equations (10) and (13), which is also verified by comparing the optimal squeezing time in Fig. 3(a)-(d). Systematically, Fig. 3(e), (f) further confirm the properties of our scheme.

It is also noticed that both the interaction strength and its sign of such an effective OAT Hamiltonian (5) are tunable. On one hand, the strength increases as the subsystem $J$ enlarges, due to $X_{\text {eff }} \propto N_{\mathrm{j}}$. On the other hand, the sign of the interaction coefficient can be flipped by simply alternating all the $\pi / 2$ rotation pulses to $-\pi / 2$ (or $3 \pi / 2$ ) rotation pulses, yielding $X_{\text {eff }}=-N_{\mathrm{j}} g^{2} \tau / 8$ and timereversal dynamics. The time-reversal untwisting dynamics is of importance to construct an interaction-based readout echo ${ }^{60,61}$, which is often applied to uncover the metrological usefulness of entangled non-Gaussian state and overcome the problem of limited atomic number resolution ${ }^{70,71}$.

\section{Generation of effective TAT squeezing}

The squeezing performance can be further enhanced to the $\mathrm{HL}$, since a more complicated pulse sequence is capable of synthesizing the TAT interaction. On this purpose, inspired by the idea in ref. ${ }^{42}$ for which the squeezing axes are alternatively changed during evolution, additional control pulses on $S$ are required since TAT interaction demands two squeezing axes, e.g., $z$-axis and $y$-axis for $S_{z}^{2}-S_{y}^{2}$. This improved scheme is illustrated in Fig. 4(a). We apply two series of rotation pulses on both subsystems $S$ and J: each period contains four $\pi / 2$ rotation pulses
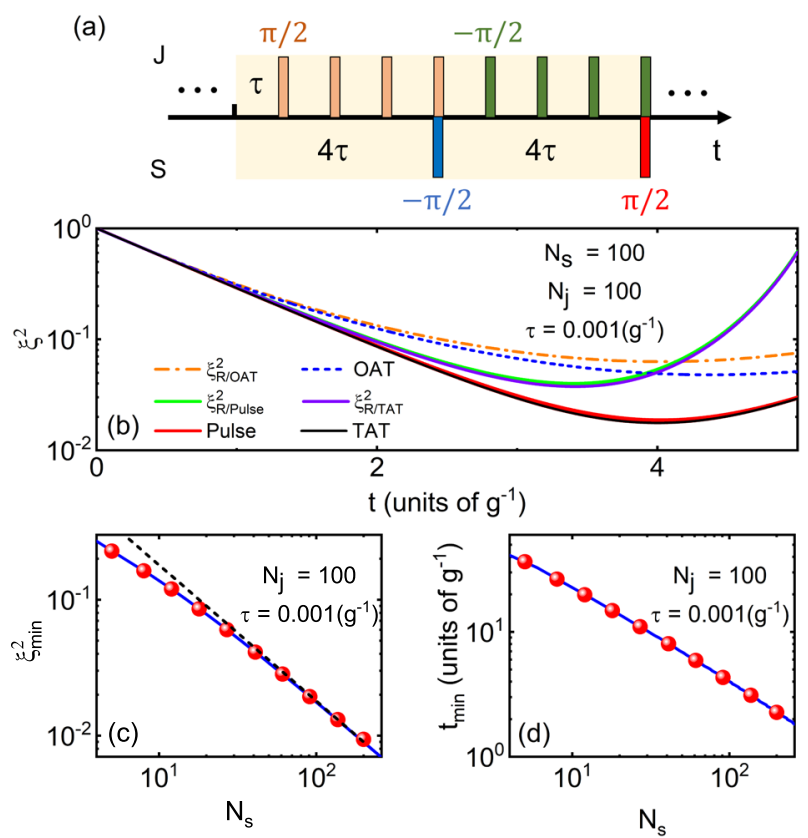

Fig. 4 Dynamic synthesis of TAT squeezing. a An illustration of the pulse sequence for the synthesis of TAT squeezing. Each period $T_{\mathrm{c}}=$ $8 \tau$, twice as long as aforementioned OAT pulse scheme, contains four $\pi / 2$ rotation pulses (orange rectangles) and four $-\pi / 2$ rotation pulses (green rectangles) imposed on subsystem $J$, and an additional pair of $\mp \pi / 2$ pulses (blue and red rectangles) imposed on subsystem $S$. b Evolution of the squeezing parameters $\xi^{2}$ for the TAT pulse scheme (red solid curve: criterion of Kitagawa and Ueda ${ }^{1}$; green solid curve: criterion of Wineland ${ }^{2,3}$ ) and for the effective TAT Hamiltonian (12) (black and blue solid curves for the two parameters), compared with the OAT dynamics (blue dashed and orange dot-dashed curves) governed by Hamiltonian (5). c and d The optimal spin squeezing parameter $\xi_{\min }^{2}$ for Kitagawa and Ueda criterion and the optimal squeezing time $t_{\min }$ as functions of the particle number $N_{\mathrm{s}}$ for the TAT pulse scheme (red solid balls), compared with the effective TAT results (blue solid curves). The black dashed line in c corresponds to $\xi_{\min }^{2}=1.8 / N_{\mathrm{s}}$. The parameters are given in each subgraph.

and four $-\pi / 2$ rotation pulses acting on subsystem $J$ with equal pulse separation $\tau$, added with a pair of $\mp \pi / 2$ rotation pulses acting on subsystem $S$ with pulse separation $4 \tau$. The evolution operator for a single period $T_{\mathrm{c}} \simeq 8 \mathrm{t}$ is given by

$U_{2}(8 \tau)=R_{\mathrm{S}}\left(\frac{\pi}{2}\right)\left[R_{J}\left(-\frac{\pi}{2}\right) U(\tau)\right]^{4} R_{\mathrm{S}}\left(-\frac{\pi}{2}\right)\left[R_{J}\left(\frac{\pi}{2}\right) U(\tau)\right]^{4}$.

The resulted effective TAT Hamiltonian is straightforward as following the derivations of the effective OAT Hamiltonian (see Supplementary Note I for details), obtained as

$H_{\text {eff }}^{\text {TAT }}=\frac{1}{2} X_{\text {eff }}\left(S_{z}^{2}-S_{y}^{2}\right)$.

Here, the effective interaction coefficient of TAT squeezing is half the case of effective OAT squeezing, i.e., $X_{\text {eff }} / 2$, while the sign of this coefficient is also flippable by merely changing the phase or areas of the rotation pulses on subsystem $J$. Since the sign of the obtained effective interaction coefficient $\chi_{\text {eff }}$ for OAT can be changed, the pulse sequence on subsystem $S$ of realizing the effective TAT squeezing is simply equally spaced pulses, much easier to realize compared with that in ref. ${ }^{42}$, as shown in Fig. 4(a). Furthermore, the effective interaction coefficient of TAT squeezing in our scheme $\left(\chi_{\text {eff }} / 2\right)$ is larger than that in ref. ${ }^{42}\left(\chi_{\text {eff }} / 3\right)$, which is more favorable for achieving the optimal spin squeezing within shorter time. 
The optimal squeezing parameter and the corresponding squeezing time for the synthesized TAT interaction is approximate to

$\xi_{\min }^{2} \simeq \frac{1.8}{N_{\mathrm{s}}}, t_{\min } \simeq \frac{8 \ln \left(4 N_{\mathrm{s}}\right)}{g^{2} \tau N_{\mathrm{j}} N_{\mathrm{s}}}$

The validity of the effective TAT Hamiltonian (12) is verified by the exact numerical simulation in Fig. 4(b)-(d). The numerical results of the pulse scheme agrees with the effective TAT squeezing, dramatically outperforming the OAT scheme. The power-law scalings of both $\xi_{\min }^{2}$ and $t_{\min }$ with respect to $N_{\mathrm{s}}$ are also the same as the TAT case, as shown in Fig. 4(c), (d). It reveals that the spin squeezing with measurement sensitivity approaches to the $\mathrm{HL}$ and its corresponding required time is much less than that of the optimal OAT squeezing. In addition, analogous to the OAT squeezing discussed above, a relatively small number of pulses can also achieve the best attainable squeezing that approaches to the optimal TAT squeezing (see Supplementary Note $\mathrm{K}$ for details).

\section{DISCUSSION}

Our scheme is robust to various noises including fluctuations of pulse areas and pulse separations. On one hand, we carry out the numerical simulation by adding Gaussian stochastic noises, i.e., assuming the fluctuating pulse areas or pulse separations are subject to Gaussian distribution with the standard deviation about five percent of the average value. The squeezing parameters of 500 independent simulations under the two types of noises are respectively shown in Fig. 5(a), (b). On the other hand, we analyze the influence of error size on the best attainable squeezing, as shown in Fig. 5(c), (d). For different pulse area or pulse separation error size, ranging from $-5 \%$ to $5 \%$, the best attainable squeezing can almost achieve the optimal squeezing of the effective OAT dynamics. Thus, it reveals that the squeezing still remains under the influence of noises, indicating the robustness of the pulse scheme. In addition, we investigate the dependence of robustness to imperfect rotations on system size. For larger particle numbers, the robustness still remains and results show that it is essentially size-independent with respect to the system (see Supplementary Note $L$ for details).

Many efforts have been made to study the decoherence of spin squeezing ${ }^{72,73}$. Particularly, an open-source library in PYTHON, the Permutational-Invariant Quantum Solver, has been developed to study different types of decoherence in open quantum system and address the robustness of various collective phenomena, including spin squeezing ${ }^{74}$. For example, in BECs, particle losses (or atomic decay) may be the major source of decoherence ${ }^{75,76}$. However, since the particle losses are the intrinsic attributes of the systems themselves instead of being caused by our pulse scheme, the particle losses have the similar influence for our scheme compared with the situation without pulses.

We note that the effective OAT or TAT squeezing can also be realized in other systems, such as nitrogen vacancy (NV) centers coupled to mechanical modes ${ }^{77-79}$. In NV centers, spin dephasing degrades the squeezing ${ }^{77}$, while it can be solved by dynamical decoupling ${ }^{80}$. Our scheme is applicable in NV centers and is compatible with dynamical decoupling, since the essence of dynamical decoupling is also applying a sequence of pulses that repetitively flip the state of the system.

In summary, we have presented a universal scheme to dynamically synthesize spin squeezing based on non-squeezing interactions and a series of rotation pulses. The non-squeezing interaction can be effectively transformed into squeezing interactions, either OAT interaction or TAT interaction. The effective interaction strengths are strongly enhanced due to the collective
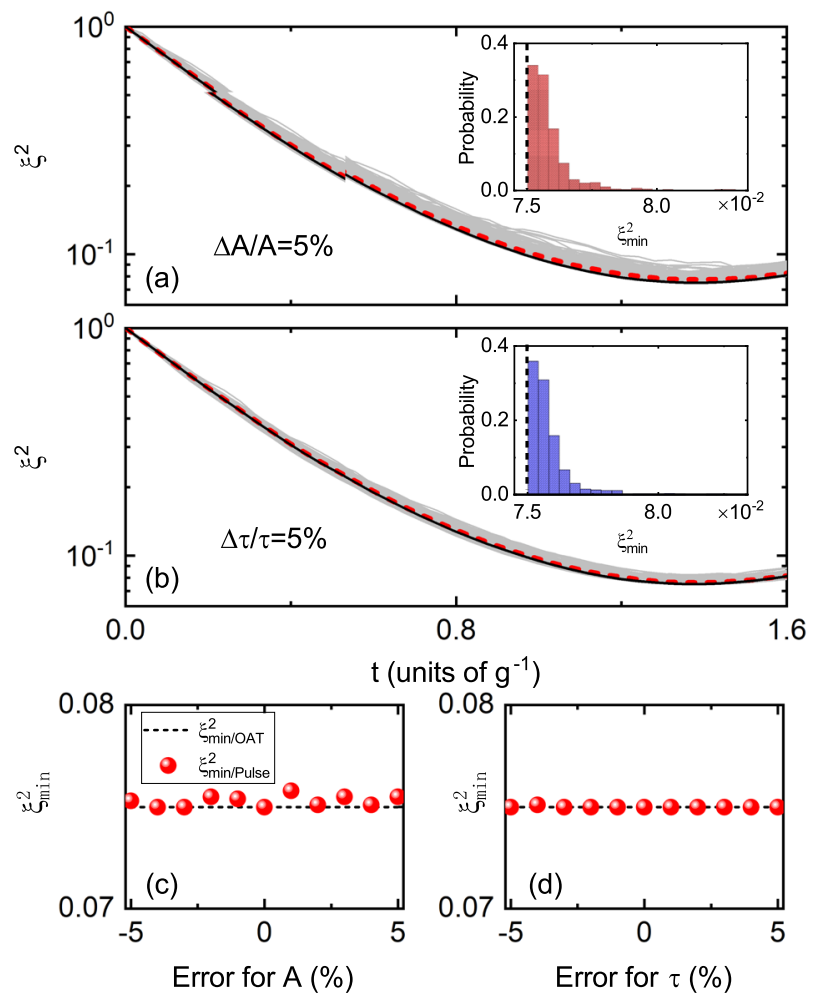

Fig. 5 Noise analysis for our scheme. Evolution of the squeezing parameter $\xi^{2}$ for $5 \%$ level of Gaussian stochastic noise adding on a the pulse area $A$ and $\mathbf{b}$ the pulse separation $\tau$. The gray curves crowded together denote the result of 500 independent simulations under noises, and the red dashed curves show their average. The black solid curves are the results without noises. Insets in $\mathbf{a}$ and $\mathbf{b}$ are the corresponding histograms of probability distribution of the consequent optimal squeezing parameter $\xi_{\text {min }}^{2}$ for 500 simulations. The ideal cases without noises are indicated by the vertical black dashed lines. $\mathbf{c}$ and $\mathbf{d}$ The best attainable spin squeezing parameter $\xi_{\min }^{2}$ as a function of the fixed relative error of pulse area $A$ and pulse separation $\tau$, respectively, for our scheme (red solid balls). The black dashed lines denote the optimal spin squeezing obtained by the effective OAT dynamics for comparison. Parameters are $N_{\mathrm{s}}=N_{\mathrm{j}}=50$ and $\tau=0.01\left(g^{-1}\right)$.

effect and their signs are also flippable, being either positive or negative, which depend on the controlled phase or area of the rotation pulses. This feature largely extends the parameter space for experiments, and enables the construction of time-reversal nonlinear interferometer with high measurement precision. The generated spin squeezing can ultimately reach the $\mathrm{HL}$ with measurement precision $\propto 1 / N_{s}$. Meanwhile, our scheme is demonstrated to be robust to fluctuating pulse areas and pulse separations. Moreover, a small number of pulses remains valid for achieving the optimal squeezing. This study provides the opportunity for realizing Heisenberg-limited spin squeezing in a large number of non-squeezing systems, which is significant for quantum metrology and quantum information science.

\section{METHODS}

\section{Calculation of the quasiprobability distribution}

First, we introduce the calculation of the quasiprobability distribution, the Husimi $Q$ function, for each subsystem. For the sake of description, we denote the quasiprobability distribution as $Q\left(\theta_{s}, \phi_{\mathrm{s}}\right)$ for subsystem $S$ and $Q$ $\left(\theta_{\mathrm{j}}, \phi_{\mathrm{j}}\right)$ for $J$. The time-dependent quantum state of the whole system is written as $|\psi(t)\rangle$ at $t$. Since the dimension of Hilbert space is $\left(N_{\mathrm{s}}+1\right)\left(N_{\mathrm{j}}+\right.$ 1 ), we project the quantum state into the product state of two arbitrary coherent spin states, i.e., $\left|\theta_{\mathrm{s}}, \phi_{\mathrm{s}}\right\rangle \otimes\left|\theta_{\mathrm{j}}, \phi_{\mathrm{j}}\right\rangle$, whose Hilbert space dimensions 
are $N_{\mathrm{s}}+1$ and $N_{\mathrm{j}}+1$, respectively. And the quasiprobability is obtained as $P=\mid\left\langle\theta_{\mathrm{s}}, \phi_{\mathrm{s}}\left|\left\langle\theta_{\mathrm{j}}, \phi_{\mathrm{j}} \mid \psi(t)\right\rangle\right|^{2}\right.$. Then we integrate the value of $P$ with respect to one pair of $(\theta, \phi)$, which is equivalent to the partial trace of the pure quantum state $|\psi(t)\rangle$, obtaining the quasiprobability distribution for one subsystem, e.g., $S$

$Q\left(\theta_{\mathrm{s}}, \phi_{\mathrm{s}}\right)=\int_{0}^{\pi} \int_{0}^{2 \pi} P d \theta_{\mathrm{j}} d \phi_{\mathrm{j}}$

It gives a representation of each subsystem on a generalized Bloch sphere with a polar angle $\theta$ and an azimuthal angle $\phi$. In the numerical calculation, for convenience, we replace the integral with a summation about these two angles in a discrete space.

\section{Calculation of the squeezing parameter}

Second, we give the brief process of calculation of the squeezing parameter $\xi^{2}=4\left(\Delta S_{\perp}\right)_{\min }^{2} / N_{s}{ }^{1}$, as mentioned previously. To calculate $\left(\Delta S_{\perp}\right)_{\min }^{2}$, we fix a direction in the perpendicular plane of the mean spin direction, might as well being denoted as $\mathbf{n}$. Therefore, in the perpendicular plane, $\left(\Delta S_{\perp}\right)^{2}$ is a function of the angle $a$, which is defined as the included angle between an arbitrary direction and the direction of $\mathbf{n}$. By making the derivative of $\left(\Delta S_{\perp}\right)^{2}$ with respect to $a$ equal to zero, i.e., $d\left(\Delta S_{\perp}\right)^{2} / d a=0$, we can obtain the minimum value of the fluctuation $\left(\Delta S_{\perp}\right)_{\min }^{2}$ with the optimal squeezing angle $a_{\mathrm{opt}}$. More detailed calculation is also given in ref. ${ }^{81}$

\section{Evolution of the squeezed spin states}

Besides, since our calculation is based on the quantum state $|\psi(t)\rangle$ at $t$, we give the description of numerical procedure of calculating $|\psi(t)\rangle$. We divide the whole evolution operator $U(t)$ into many small equal parts $U(\delta t)$, denoting a segment of free evolution under the Hamiltonian (1) for a very short time $\delta t(\delta t \ll \tau)$. Given the initial coherent spin state $|\psi\rangle$, we apply the individual evolution operator $U(\delta t)$, together with the rotation operators $R_{\mathrm{S}}( \pm \pi / 2)$ or $R_{\mathrm{J}}( \pm \pi / 2)$ at appropriate time instants, repeatedly on the evolved state and can approximately obtain the time-dependent state. As a result, the evolution of quantum state and squeezing parameter are obtained.

\section{DATA AVAILABILITY}

The authors declare that all data supporting the findings of this study are available within the article or from the corresponding author upon reasonable request.

Received: 15 January 2021; Accepted: 13 November 2021; Published online: 09 December 2021

\section{REFERENCES}

1. Kitagawa, M. \& Ueda, M. Squeezed spin states. Phys. Rev. A 47, 5138-5143 (1993).

2. Wineland, D. J., Bollinger, J. J., Itano, W. M., Moore, F. L. \& Heinzen, D. J. Spin squeezing and reduced quantum noise in spectroscopy. Phys. Rev. A 46, R6797-R6800 (1992).

3. Wineland, D. J., Bollinger, J. J., Itano, W. M. \& Heinzen, D. J. Squeezed atomic states and projection noise in spectroscopy. Phys. Rev. A 50, 67-88 (1994).

4. Ma, J., Wang, X., Sun, C. \& Nori, F. Quantum spin squeezing. Phys. Rep. 509, 89-165 (2011).

5. Meiser, D., Ye, J. \& Holland, M. J. Spin squeezing in optical lattice clocks via latticebased QND measurements. N. J. Phys. 10, 073014 (2008).

6. André, A., Sørensen, A. S. \& Lukin, M. D. Stability of atomic clocks based on entangled atoms. Phys. Rev. Lett. 92, 230801 (2004).

7. Leroux, I. D., Schleier-Smith, M. H. \& Vuletić, V. Implementation of cavity squeezing of a collective atomic spin. Phys. Rev. Lett. 104, 073602 (2010).

8. Gross, C., Zibold, T., Nicklas, E., Estève, J. \& Oberthaler, M. K. Nonlinear atom interferometer surpasses classical precision limit. Nat. (Lond.) 464, 1165-1169 (2010).

9. Riedel, M. F. et al. Atom-chip-based generation of entanglement for quantum metrology. Nat. (Lond.) 464, 1170-1173 (2010).

10. Kaubruegger, R. et al. Variational spin-squeezing algorithms on programmable quantum sensors. Phys. Rev. Lett. 123, 260505 (2019).

11. Sørensen, A., Duan, L.-M., Cirac, J. I. \& Zoller, P. Many-particle entanglement with Bose CEinstein condensates. Nature 409, 63-66 (2001).
12. Tóth, G., Knapp, C., Gühne, O. \& Briegel, H. J. Spin squeezing and entanglement. Phys. Rev. A 79, 042334 (2009).

13. Korbicz, J. K., Cirac, J. I. \& Lewenstein, M. Spin squeezing inequalities and entanglement of N Qubit States. Phys. Rev. Lett. 95, 120502 (2005).

14. Korbicz, J. K. et al. Generalized spin-squeezing inequalities in $\mathrm{N}$-qubit systems: theory and experiment. Phys. Rev. A 74, 052319 (2006).

15. Hyllus, P., Pezzé, L., Smerzi, A. \& Tóth, G. Entanglement and extreme spin squeezing for a fluctuating number of indistinguishable particles. Phys. Rev. A 86, 012337 (2012).

16. Sørensen, A. S. \& Mølmer, K. Entanglement and Extreme Spin Squeezing. Phys. Rev. Lett. 86, 4431-4434 (2001).

17. Perlin, M. A., Qu, C. \& Rey, A. M. Spin Squeezing with Short-Range Spin-Exchange Interactions. Phys. Rev. Lett. 125, 223401 (2020).

18. Orzel, C., Tuchman, A. K., Fenselau, M. L., Yasuda, M. \& Kasevich, M. A. Squeezed states in a bose-einstein condensate. Science 291, 2386-2389 (2001).

19. Estève, J., Gross, C., Weller, A., Giovanazzi, S. \& Oberthaler, M. K. Squeezing and entanglement in a Bose-Einstein condensate. Nature 455, 1216-1219 (2008).

20. Fadel, M., Zibold, T., Décamps, B. \& Treutlein, P. Spatial entanglement patterns and Einstein-podolsky-rosen steering in bose-Einstein condensates. Science $\mathbf{3 6 0}$, 409-413 (2018).

21. Hammerer, K., Sørensen, A. S. \& Polzik, E. S. Quantum interface between light and atomic ensembles. Rev. Mod. Phys. 82, 1041-1093 (2010).

22. $\mathrm{Yu}$, L. et al. Creating a tunable spin squeezing via a time-dependent collective atom-photon coupling. Phys. Rev. A 89, 023838 (2014).

23. Evrard, A. et al. Enhanced magnetic sensitivity with non-gaussian quantum fluctuations. Phys. Rev. Lett. 122, 173601 (2019)

24. Zhang, Y.-L., Zou, C.-L., Zou, X.-B., Jiang, L. \& Guo, G.-C. Detuning-enhanced cavity spin squeezing. Phys. Rev. A 91, 033625 (2015).

25. Qin, W., Chen, Y. H., Wang, X., Miranowicz, A. \& Nori, F. Strong spin squeezing induced by weak squeezing of light inside a cavity. Nanophotonics 9, 4853-4868 (2020).

26. Kuzmich, A., Mølmer, K. \& Polzik, E. S. Spin squeezing in an ensemble of atoms illuminated with squeezed light. Phys. Rev. Lett. 79, 4782-4785 (1997).

27. Hald, J., Sørensen, J. L., Schori, C. \& Polzik, E. S. Spin squeezed atoms: a macroscopic entangled ensemble created by light. Phys. Rev. Lett. 83, 1319-1322 (1999).

28. Vernac, L., Pinard, M. \& Giacobino, E. Spin squeezing in two-level systems. Phys. Rev. A 62, 063812-063811 (2000).

29. Fleischhauer, M. \& Gong, S. Stationary source of nonclassical or entangled atoms. Phys. Rev. Lett. 88, 070404 (2002).

30. Kuzmich, A., Mandel, L. \& Bigelow, N. P. Generation of spin squeezing via continuous quantum nondemolition measurement. Phys. Rev. Lett. 85, 1594-1597 (2000).

31. Kuzmich, A., Bigelow, N. P. \& Mandel, L. Atomic quantum non-demolition measurements and squeezing. Europhys. Lett. 42, 481-486 (1998).

32. Kuzmich, A. et al. Quantum nondemolition measurements of collective atomic spin. Phys. Rev. A 60, 2346-2350 (1999).

33. Takano, T., Fuyama, M., Namiki, R. \& Takahashi, Y. Spin squeezing of a cold atomic ensemble with the nuclear spin of one-half. Phys. Rev. Lett. 102, 033601 (2009).

34. Inoue, R., Tanaka, S.-I.-R., Namiki, R., Sagawa, T. \& Takahashi, Y. Unconditional quantum-noise suppression via measurement-based quantum feedback. Phys. Rev. Lett. 110, 163602 (2013).

35. Schleier-Smith, M. H., Leroux, I. D. \& Vuletić, V. States of an ensemble of two-level atoms with reduced quantum uncertainty. Phys. Rev. Lett. 104, 073604 (2010).

36. Chen, Z., Bohnet, J. G., Sankar, S. R., Dai, J. \& Thompson, J. K. Conditional spin squeezing of a large ensemble via the vacuum rabi splitting. Phys. Rev. Lett. 106, 133601 (2011).

37. Bao, H. et al. Spin squeezing of 1011 atoms by prediction and retrodiction measurements. Nature 581, 159-163 (2020).

38. Hosten, O., Engelsen, N. J., Krishnakumar, R. \& Kasevich, M. A. Measurement noise 100 times lower than the quantum-projection limit using entangled atoms. Nature 529, 505-508 (2016).

39. Pezzè, L., Smerzi, A., Oberthaler, M. K., Schmied, R. \& Treutlein, P. Quantum metrology with nonclassical states of atomic ensembles. Rev. Mod. Phys. 90, 1609.01609 (2018).

40. Rossi, M. A., Albarelli, F., Tamascelli, D. \& Genoni, M. G. Noisy quantum metrology enhanced by continuous nondemolition measurement. Phys. Rev. Lett. 125, 200505 (2020).

41. Chen, F., Chen, J.-J., Wu, L.-N., Liu, Y.-C. \& You, L. Extreme spin squeezing from deep reinforcement learning. Phys. Rev. A 100, 041801(R) (2019).

42. Liu, Y. C., Xu, Z. F., Jin, G. R. \& You, L. Spin squeezing: Transforming one-axis twisting into two-axis twisting. Phys. Rev. Lett. 107, 013601 (2011).

43. Zhang, J. Y., Zhou, X. F., Guo, G. C. \& Zhou, Z. W. Dynamical spin squeezing via a higher-order Trotter-Suzuki approximation. Phys. Rev. A 90, 013604 (2014). 
44. Huang, W., Zhang, Y.-L., Zou, C.-L., Zou, X.-B. \& Guo, G.-C. Two-axis spin squeezing of two-component Bose-Einstein condensates via continuous driving. Phys. Rev. A 91, 043642 (2015).

45. Shen, C. \& Duan, L.-M. Efficient spin squeezing with optimized pulse sequences. Phys. Rev. A 87, 051801 (2013).

46. Zhang, Y. C., Zhou, X. F., Zhou, X., Guo, G. C. \& Zhou, Z. W. Cavity-assisted singlemode and two-mode spin-squeezed states via phase-locked atom-photon coupling. Phys. Rev. Lett. 118, 083604 (2017).

47. Hu, J. et al. Vacuum spin squeezing. Phys. Rev. A 96, 1-5 (2017).

48. Helmerson, K. \& You, L. Creating massive entanglement of bose-einstein condensed atoms. Phys. Rev. Lett. 87, 170402 (2001).

49. Bohnet, J. G. et al. Quantum spin dynamics and entanglement generation with hundreds of trapped ions. Science 352, 1297-1301 (2016).

50. Lu, Y. et al. Global entangling gates on arbitrary ion qubits. Nature 572, 363-367 (2019).

51. Figgatt, $C$. et al. Parallel entangling operations on a universal ion-trap quantum computer. Nature 572, 368-372 (2019).

52. Song, C. et al. 10-qubit entanglement and parallel logic operations with a superconducting circuit. Phys. Rev. Lett. 119, 180511 (2017).

53. Song, C. et al. Generation of multicomponent atomic schrödinger cat states of up to 20 qubits. Science 365, 574-577 (2019).

54. $\mathrm{Xu}, \mathrm{K}$. et al. Probing dynamical phase transitions with a superconducting quantum simulator. Sci. Adv. 6, eaba4935 (2020).

55. Borregaard, J., Davis, E. J., Bentsen, G. S., Schleier-Smith, M. H. \& Sørensen, A. S. One- and two-axis squeezing of atomic ensembles in optical cavities. N. J. Phys. 19, 093021 (2017).

56. Macrì, V., Nori, F., Savasta, S. \& Zueco, D. Spin squeezing by one-photon-twoatom excitation processes in atomic ensembles. Phys. Rev. A 101, 053818 (2020).

57. Takeuchi, M. et al. Spin squeezing via one-axis twisting with coherent light. Phys. Rev. Lett. 94, 023003 (2005)

58. Wang, M. et al. Two-axis-twisting spin squeezing by multipass quantum erasure. Phys. Rev. A 96, 013823 (2017).

59. Trail, C. M., Jessen, P. S. \& Deutsch, I. H. Strongly enhanced spin squeezing via quantum control. Phys. Rev. Lett. 105, 193602 (2010)

60. Linnemann, D. et al. Quantum-enhanced sensing based on time reversal of nonlinear dynamics. Phys. Rev. Lett. 117, 013001 (2016).

61. Davis, E., Bentsen, G. \& Schleier-Smith, M. Approaching the Heisenberg limit without single-particle detection. Phys. Rev. Lett. 116, 053601 (2016).

62. Gärttner, M. et al. Measuring out-of-time-order correlations and multiple quantum spectra in a trapped-ion quantum magnet. Nat. Phys. 13, 781-786 (2017).

63. $\mathrm{Li}$, J. et al. Measuring out-of-time-order correlators on a nuclear magnetic resonance quantum simulator. Phys. Rev. X 7, 031011 (2017).

64. Hummel, Q., Geiger, B., Urbina, J. D. \& Richter, K. Reversible quantum information spreading in many-body systems near criticality. Phys. Rev. Lett. 123, 160401 (2019).

65. Nie, X. et al. Experimental observation of equilibrium and dynamical quantum phase transitions via out-of-time-ordered correlators. Phys. Rev. Lett. 124, 250601 (2020).

66. Jing, Y., Fadel, M., Ivannikov, V. \& Byrnes, T. Split spin-squeezed Bose CEinstein condensates. N. J. Phys. 21, 093038 (2019).

67. Fröhlich, $\mathrm{H}$. Theory of the superconducting state. i. the ground state at the absolute zero of temperature. Phys. Rev. 79, 845-856 (1950).

68. Nakajima, S. Perturbation theory in statistical mechanics. Adv. Phys. 4, 363-380 (1955).

69. Schleier-Smith, M. H., Leroux, I. D. \& Vuletić, V. Squeezing the collective spin of a dilute atomic ensemble by cavity feedback. Phys. Rev. A 81, 021804 (2010).

70. Haine, S. A. Using interaction-based readouts to approach the ultimate limit of detection-noise robustness for quantum-enhanced metrology in collective spin systems. Phys. Rev. A 98, 030303 (2018).

71. Nolan, S. P., Szigeti, S. S. \& Haine, S. A. Optimal and robust quantum metrology using interaction-based readouts. Phys. Rev. Lett. 119, 193601 (2017).

72. Wang, X., Miranowicz, A., Liu, Y.-x, Sun, C. P. \& Nori, F. Sudden vanishing of spin squeezing under decoherence. Phys. Rev. A 81, 022106 (2010).

73. Yin, X., Ma, J., Wang, X. \& Nori, F. Spin squeezing under non-markovian channels by the hierarchy equation method. Phys. Rev. A 86, 012308 (2012).

74. Shammah, N., Ahmed, S., Lambert, N., De Liberato, S. \& Nori, F. Open quantum systems with local and collective incoherent processes: efficient numerical simulations using permutational invariance. Phys. Rev. A 98, 063815 (2018).
75. Li, Y., Castin, Y. \& Sinatra, A. Optimum spin squeezing in bose-einstein condensates with particle losses. Phys. Rev. Lett. 100, 210401 (2008).

76. Li, Y., Treutlein, P., Reichel, J. \& Sinatra, A. Spin squeezing in a bimodal condensate: spatial dynamics and particle losses. Eur. Phys. J. B 68, 365-381 (2009).

77. Bennett, S. D. et al. Phonon-induced spin-spin interactions in diamond nanostructures: application to spin squeezing. Phys. Rev. Lett. 110, 156402 (2013).

78. Ma, Y. H., Zhang, X. F., Song, J. \& Wu, E. Bistability and steady-state spin squeezing in diamond nanostructures controlled by a nanomechanical resonator. Ann. Phys. (N. Y). 369, 36-44 (2016).

79. Xia, K. \& Twamley, J. Generating spin squeezing states and Greenberger-HorneZeilinger entanglement using a hybrid phonon-spin ensemble in diamond. Phys. Rev. B 94, 1-8 (2016).

80. Bar-Gill, N., Pham, L. M., Jarmola, A., Budker, D. \& Walsworth, R. L. Solid-state electronic spin coherence time approaching one second. Nat. Commun. 4 1211.7094 (2013)

81. Jin, G.-R., Liu, Y.-C. \& Liu, W.-M. Spin squeezing in a generalized one-axis twisting model. N. J. Phys. 11, 073049 (2009).

\section{ACKNOWLEDGEMENTS}

This work is supported by the Key-Area Research and Development Program of Guangdong Province (Grant No. 2019B030330001), the National Natural Science Foundation of China (NSFC) (Grant Nos. 92050110, 91736106, 11674390, and 91836302), and the National Key R\&D Program of China (Grants No. 2018YFA0306504).

\section{AUTHOR CONTRIBUTIONS}

Y.L. conceived the idea; L.H. and Y.L. carried out the calculations and wrote the paper All authors contributed to the discussion of the results; Y.L. supervised the project.

\section{COMPETING INTERESTS}

The authors declare no competing interests.

\section{ADDITIONAL INFORMATION}

Supplementary information The online version contains supplementary material available at https://doi.org/10.1038/s41534-021-00505-z.

Correspondence and requests for materials should be addressed to Yong-Chun Liu.

Reprints and permission information is available at http://www.nature.com/ reprints

Publisher's note Springer Nature remains neutral with regard to jurisdictional claims in published maps and institutional affiliations.

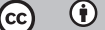

Open Access This article is licensed under a Creative Commons Attribution 4.0 International License, which permits use, sharing, adaptation, distribution and reproduction in any medium or format, as long as you give appropriate credit to the original author(s) and the source, provide a link to the Creative Commons license, and indicate if changes were made. The images or other third party material in this article are included in the article's Creative Commons license, unless indicated otherwise in a credit line to the material. If material is not included in the article's Creative Commons license and your intended use is not permitted by statutory regulation or exceeds the permitted use, you will need to obtain permission directly from the copyright holder. To view a copy of this license, visit http://creativecommons. org/licenses/by/4.0/.

(c) The Author(s) 2021 